\title{
The variation of reconnection rate at the dayside magnetopause and cusp ion precipitation
}

Article

Published Version

Lockwood, M. and Smith, M. F. (1992) The variation of reconnection rate at the dayside magnetopause and cusp ion precipitation. Journal of Geophysical Research, 97 (A10). pp. 14841-14847. ISSN 0148-0227 doi: https://doi.org/10.1029/92JA01261 Available at https://centaur.reading.ac.uk/38830/

It is advisable to refer to the publisher's version if you intend to cite from the work. See Guidance on citing.

Published version at: http://dx.doi.org/10.1029/92JA01261

To link to this article DOI: http://dx.doi.org/10.1029/92JA01261

All outputs in CentAUR are protected by Intellectual Property Rights law, including copyright law. Copyright and IPR is retained by the creators or other copyright holders. Terms and conditions for use of this material are defined in the End User Agreement.

www.reading.ac.uk/centaur 
Central Archive at the University of Reading

Reading's research outputs online 


\title{
The Variation of Reconnection Rate at the Dayside Magnetopause and Cusp Ion Precipitation
}

\author{
M. LOCKWOOD ${ }^{1}$
}

Rutherford Appleton Laboratory, Chilton, Didcot, United Kingdom

\author{
M. F. SMITH \\ Laboratory for Extraterrestrial Physics, NASA Goddard Space Flight Center, Greenbelt, Maryland
}

\begin{abstract}
A method is presented which allows estimation of the variation of the rate of magnetic reconnection at the dayside magnetopause. This is achieved using observations of the cusp particle precipitation made by low-altitude polar-orbiting spacecraft. In this paper we apply the technique to a previously published example of a cusp intersection by the DMSP F7 satellite. It is shown that the cusp signature in this case was produced by three separate bursts of reconnection which were of the order of $10 \mathrm{~min}$ apart, each lasting roughly $1 \mathrm{~min}$. This is similar to the variation of reconnection rate which is required to explain typical flux transfer event signatures at the magnetopause.
\end{abstract}

\section{INTRODUCTION}

In this paper we discuss the implications of the dispersion of the precipitating magnetosheathlike ions in the region termed the "cusp." In particular, we investigate the rate of reconnection at the dayside magnetopause and the "pulsating cusp" concept of Smith and Lockwood [1990]. By considering a pass of the DE 2 satellite, Lockwood and Smith [1989] concluded that the cusp particle precipitation observed was consistent with the ionospheric signature of a burst of reconnection at the dayside magnetopause. Such reconnection bursts had long been invoked as a cause of transient particle and field signatures near the dayside magnetopause, which were therefore termed "flux transfer events" (FTEs) [Russell and Elphic, 1978, 1979; Haerendel et al., 1978; Paschmann et al., 1982; Berchem and Russell, 1984; Rijnbeek et al., 1984; Farrugia et al., 1987a, b, 1988; Lockwood, 1991a].

The suggestion of Lockwood and Smith [1989] was based on the convective flows, inferred filamentary field-aligned currents, and precipitating ion and electron characteristics. Newell [1990] objected that the extended longitudinal width of the cusp occurrence (covering several hours of MLT) was not consistent with the cusp being a nearly circular signature of a flux transfer event, as predicted by Southwood [1985, 1987] for the Russell and Elphic [1978, 1979] FTE model. However, Lockwood and Smith [1990] pointed out that such longitudinal elongation was indeed consistent with the cusp being the ionospheric signature of an FTE, provided either the Southwood et al. [1988]/Scholer [1988] single elongated $\mathrm{X}$ line model or, alternatively, the Lee and $F u$ [1985] multiple elongated $\mathrm{X}$ line model was adopted.

The Southwood et al. [1988]/Scholer [1988] model predicts that an FTE signature on the magnetopause is formed by a short-lived burst of enhanced reconnection at a single sub-

\footnotetext{
${ }^{1}$ Also visiting at Imperial College, London.

Copyright 1992 by the American Geophysical Union.
}

Paper number 92JA01261.

0148-0227/92/92JA-01261\$05.00 solar $\mathrm{X}$ line. There are two major pieces of evidence in favor of this concept, as opposed to the other FTE models which invoke reconnection: first, it explains a layer of bidirectionally streaming electrons found on the inner edge of magnetopause FTE signatures [Scudder et al., 1984; Farrugia et al., 1988]; second, it is consistent with the elongation of dayside transient aurorae and flow bursts observed in the cusp region, which repeat with the same range of periods as magnetopause FTEs [Lockwood et al., 1990b] and move in a manner consistent with their being on newly opened field lines. Because most of the cusp precipitation is thought to be on freshly reconnected field lines [Rosenbauer et al., 1975; Reiff et al., 1977; Hill, 1979; Cowley et al., 1991a], Smith and Lockwood [1990] pointed out that the reconnection rate variations thought to be responsible for magnetopause FTEs should also cause the cusp to vary on time scales of about 2-20 min. This "pulsating cusp" model is a general one, reducing to a steady state cusp in the one limit of constant magnetopause reconnection rate, while predicting the cusp to be a series of discrete events in the other limit of reconnection which occurs entirely in a series of bursts.

Cowley et al. [1991 b] and Smith et al. [1992] have pointed out the importance to the pulsating cusp concept of recent empirical and theoretical work on how ionospheric flow is excited by dayside reconnection. The observations in question were of the rapid response of dayside flows to the north/south component of the interplanetary magnetic field (IMF) immediately upstream from the magnetopause, in particular, those using the European Incoherent Scatter (EISCAT) radar with the AMPTE (Active Magnetospheric Particle Tracer Explorers) UKS and IRM satellites [Lockwood et al., 1990a; Cowley and Lockwood, 1992]. In particular, the important inference for the pulsating cusp concept is that flows are excited in the ionosphere by a burst of magnetopause reconnection, such that for moderate and small IMF $B_{y}$, the patch of newly opened magnetic flux produced by a subsequent burst will lie immediately equatorward of the patch produced by the first burst. In other words, the patches of newly opened flux produced by successive bursts of reconnection are spatially contiguous. A slightly different situation would apply to large $B_{y}$ condi- 
tions, where the new patch would form at a different MLT from the location of the older one but would then move zonally under magnetic tension to join it. Cowley et al. and Smith et al. also point out that the ion precipitation characteristics would differ in the patches, depending on the time elapsed since they were reconnected. This would result in a discontinuous change in the ion energy at the boundary between the patches. Indeed, in the "discrete event" limit of the pulsating cusp model, the changes in energy are instantaneous. In cases where the reconnection rate variations are superposed on a background level, the ion energy changes at the boundaries are more gradual, but still more rapid than those within each patch (which result from the nonzero time taken to reconnect each patch).

Independent of these theoretical predictions, Newell and Meng [1991] have recently published examples of cusp ion spectrograms from the DMSP F7 satellite which show discontinuous changes in the ion energy-time dispersion. These are exactly of the kind predicted by Cowley et al. and Smith et al. This paper discusses these DMSP F7 observations in terms of variations in reconnection rate (i.e., by definition, flux transfer events). This interpretation is significantly different from that presented by Newell and Meng which is in terms of spatial, rather than temporal, structure. In section 2 a method is presented to allow the reconnection rate to be computed from the ion energy-time spectrogram. The method is then applied in section 3 to one of the examples given by Newell and Meng, and the results are discussed in section 4 .

\section{METHOD}

We here consider only cusp ions which precipitate into the ionosphere and are detected by polar-orbiting satellites at low altitudes (i.e., below about $1500 \mathrm{~km}$ ). Previous studies [e.g., Rosenbauer et al., 1975; Reiff et al., 1977; Menietti and Burch, 1988] have assumed that the bulk of the cusp ions move adiabatically, with little or no pitch angle scattering, from the outer magnetosphere to the ionosphere. This assumption has successfully explained the well-known pitch angle-energy and energy-latitude dispersions of cusp ions and is also adopted here. Hence we are only concerned with particles which are very close to zero pitch angle at great altitudes: all others mirror in the converging magnetic field lines before reaching the ionosphere. We consider only ions with exactly zero pitch angle at all altitudes; their energy, $E_{\|}$, is given by

$$
E_{\|}=m v_{\|}^{2} / 2
$$

where $m$ is the ion mass and $v_{\|}$is its field-aligned velocity. We note that particles having very close to, but not exactly, zero pitch angle at great altitudes may still reach the satellite, where they would have larger pitch angles. Such ions will have a slightly higher energy, for the same time of flight, than the zero pitch angle ions we consider. Hence such ions are not relevant if we consider the minimum ion energy observed by the satellite.

Reiff et al. [1977] deduced that cusp particle precipitation was predominantly on "newly opened" field lines at the sunward edge of the polar cap. The evidence from magnetopause observations for southward IMF is that reconnection always takes place at low latitudes. This follows both a)

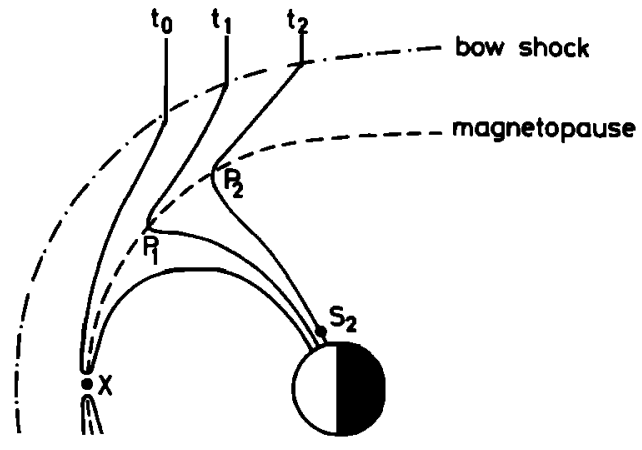

b)

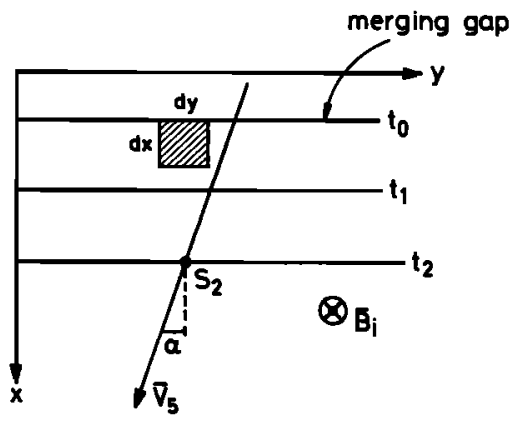

Fig. 1. (a) The evolution of a newly opened field line which is reconnected at a subsolar magnetopause at a time $t_{0}$. (b) The motion of the ionospheric feet of field lines reconnected at time $t_{0}$ in the northern hemisphere. The vector $\mathbf{V}_{s}$ shows the satellite motion.

from the observed accelerated flows [Cowley, 1982; Gosling et al., 1990] and from the motion and polarities of FTEs [Daly et al., 1984; Southwood et al., 1986]. Figure $1 a$ shows the evolution of a single such field line after it is reconnected at an $X$ line in the vicinity of the subsolar magnetopause ( $X$ in Figure $1 a$ ), at a time $t_{0}$. At subsequent times, $t_{1}$ and $t_{2}$, the field line will thread the magnetopause at higher magnetic latitudes (at points $P_{1}$ and $P_{2}$ in Figure $1 a$ ) as it is moved antisunward by magnetic tension and the magnetosheath flow. The first ions to cross the magnetopause, and hence be injected onto the newly opened field line, do so at the time $t_{0}$ (and at a location very close to $X$ ). Hence at a time $t_{2}$ a satellite at $S_{2}$ cannot observe ions with a time of flight greater than $\left(t_{2}-t_{0}\right)$, and therefore there is a minimum "cutoff" ion energy $\left(E_{\|}=E_{i c}\right)$. At the same time the satellite will observe ions of greater energy $\left(E_{\|}>E_{i c}\right)$, these having crossed the magnetopause at points such as $\mathrm{P}_{1}$, i.e., at time $t_{1}$, and hence they have a lower time of flight, $\left(t_{2}\right.$ $\left.-t_{1}\right)$.

These considerations show that the lowest-energy ions observed at low altitudes at any one instant were injected across the magnetopause at the time and place that reconnection of that field line occurred. At time $t_{0}$ the field line is opened; and hence, by definition of reconnection, it subsequently threads the magnetopause, and ions from the magnetosheath cross the magnetopause into the magnetosphere. The first ions injected in this way travel the distance $d$ from the $\mathrm{X}$ line to the satellite $S_{2}$. All magnetosheath ions which cross the dayside magnetopause are accelerated in the Earth's frame of reference, such that their $E_{\|}$can be increased by a factor of up to about 2 or 3 . This acceleration occurs as the ion crosses the magnetopause and is due to the tension force arising from the curvature of the newly opened 
field line at the magnetopause, as shown in Figure 1a. Ions which enter at the later evolution times of the field line (and hence at higher magnetic latitudes) are less strongly accelerated due to the straightening of the field line. Indeed, antisunward of the magnetic cusp the ions are decelerated as they cross the magnetopause [Hill, 1979]. Cowley [1982] has discussed how the acceleration due to the contracting newly opened field lines moving along the magnetopause current layer (sunward of the magnetic cusp) produces a characteristic D-shaped ion distribution function, which has recently been confirmed using AMPTE observations of injected ions [Smith and Rodgers, 1991; Fuselier et al., 1991].

To first order, the ions are therefore accelerated as they are injected across the magnetopause close to $X$ and subsequently precipitate adiabatically along the distance $d$ to the ionosphere. However, we can generalize to allow for any subsequent field-parallel acceleration by defining an equivalent (or "virtual") distance $d$ ' such that the actual time of flight of the ions, $\tau$, is given by

$$
\tau=d^{\prime} / v_{\|}=d^{\prime}\left(2 E_{\|} / m\right)^{-1 / 2}
$$

In other words, if the ions originated from a source and then moved adiabatically (with constant energy equal to that observed at the satellite, $\left.E_{\|}\right)$, then that source would need to be a distance $d^{\prime}$ from the satellite to give the same time of flight as for ions which had an injection distance $d$ but underwent some subsequent parallel acceleration. In general, $d^{\prime}$ will exceed $d$, but because subsequent parallel acceleration is small, $d^{\prime}$ and $d$ will generally be similar.

If ions with the lower cutoff energy are observed by the satellite at a time $t_{s}$, the time that the field line was reconnected is

$$
t_{0}=t_{s}-\tau=t_{s}-d^{\prime}\left(2 E_{i c} / m\right)^{-1 / 2}
$$

Consider now Figure $1 b$, which views the northern hemisphere cusp region in the ionosphere from above. The $y$ axis is parallel to the ionospheric signature of the reconnection $\mathrm{X}$ line (the so-called "merging gap"), and the $x$ axis is the (roughly) northward normal to the merging gap. The $x-y$ frame is here considered stationary in the frame of the Earth. The positions of the foot of the newly opened field line studied in Figure $1 a$ are shown for the times $t_{0}, t_{1}$, and $t_{2}$. It should be noted that this figure portrays the idealized situation where both the reconnection rate and the subsequent motion of the opened field lines are independent of $y$. By definition, the position for $t_{0}$ marks the merging gap location. In addition, a path of a polar-orbiting satellite is shown in Figure $1 b$. This satellite is moving with a velocity $V_{s}$ in the $x-y$ frame, which is defined as positive if it has a positive $x$ component. The angle between $\mathrm{V}_{s}$ and the $x$ axis is denoted by $\alpha$. Hence

$$
d x / d t_{s}=V_{s} \cos \alpha
$$

If we differentiate (3) with respect to $x$, we obtain

$$
\begin{aligned}
& d t_{0} / d x=d t_{s} / d x+\left(d^{\prime} / 2\right)(m / 2)^{1 / 2} E_{i c}^{-3 / 2} d E_{i c} / d x \\
& =\left(V_{s} \cos \alpha\right)^{-1}\left\{1+\left(d^{\prime} / 2\right)(m / 2)^{1 / 2} E_{i c}^{-3 / 2} d E_{i c} / d t_{s}\right\}
\end{aligned}
$$

Consider an element of length of the merging gap $d y$. The field lines which lie a small distance $d x$ poleward of the merging gap were reconnected at a time $\left(t_{0}-d t_{0}\right)$, slightly earlier than those at the merging gap which are reconnected at the time $t_{0}$. Note that this statement is independent of any motion of the merging gap. Hence by Faraday's law the rate at which flux is opened at the merging gap element $d y$ is

$$
B_{i} d x d y / d t_{0}=E_{y} d y=E_{y}^{\prime} d y^{\prime}
$$

where $B_{i}$ is the ionospheric field strength and $E_{y}$ is the electric field along the merging gap (in its own rest frame). By definition of the merging gap, the flux transfer rate across the segment $d y$ of the merging gap (i.e., the voltage $E_{y} d y$ ) is equal to the flux transfer rate (voltage) across the corresponding segment of the magnetopause $X$ line, $E_{y}^{\prime} d y^{\prime}$, where $E_{y}^{\prime}$ is the reconnection rate and $d y^{\prime}$ is the length of the $\mathrm{X}$ line segment which maps down the magnetic field lines to the length $d y$ of the merging gap in the ionosphere. From equations (5) and (6) we have

$$
E_{y}=\left(B_{i} V_{s} \cos \alpha\right)\left\{1+\left(d^{\prime} / 2\right)(m / 2)^{1 / 2} E_{i c}^{-3 / 2} d E_{i c} / d t_{s}\right\}^{-1}
$$

and the magnetopause reconnection rate $E_{y}^{\prime}$ is given by

$$
E_{y}^{\prime}=\left(d y / d y^{\prime}\right) E_{y}
$$

From a cusp ion spectrogram we can determine the minimum energy, $E_{i c}$, and its time derivative, $d E_{i c} / d t_{s}$. We also know the satellite speed $V_{s}$. We assume that the ions are protons (i.e., $m$ is $1 \mathrm{amu}$ ), and the ionospheric field $B_{i}$ is taken to be $5 \times 10^{-5} \mathrm{~T}$. Hence the only variables in equation (7) which are not known are the merging gap orientation angle, $\alpha$, and the virtual injection distance, $d^{\prime}$. In the next section we evaluate $E_{y}$ using assumed values for $\alpha$ and $d^{\prime}$. Using equation (8), we also discuss the implications for the reconnection rate for a given mapping factor $\left(d y / d y^{\prime}\right)$.

Lastly, one should note that the method inherently assumes that the same ion spectrum is injected onto each newly opened field line as it evolves over the dayside magnetopause. There are a number of reasons why this may not always be the case. For example, solar wind parameters may vary and may cause the magnetosheath ion populations to change. In addition, changes in the IMF orientation may alter the degree of acceleration of the ions as they cross the dayside magnetopause. Hence we note that variations in the reconnection rate are not the only way to induce ion energy changes in the cusp.

\section{Application to a Satellite Pass Through the Cusp}

As we discussed in the introduction, Newell and Meng [1991] have recently presented a number of passes of the DMSP F7 satellite through the cusp, which yield steplike discontinuities in the ion energy dispersion signature. Independent of these observations, Smith et al. [1992] and Cowley et al. [1991b] had predicted such signatures by applying the "pulsating cusp" model [Lockwood and Smith, 1989, 1990; Smith and Lockwood, 1990].

In order to investigate the implications of the observed jumps in ion energy in further detail, the equations derived in section 2 are here applied to the example given by Newell and Meng [1991] in their Figure $1(b)$. This example is employed because the spectrogram shows two clear jumps in the ion dispersion signature, at 1320:25 UT and 1321:7.5 UT. As discussed by Smith et al. [1992] and Cowley et al. [1991b], such jumps could arise from two periods when little reconnection took place, in between three bursts of rapid reconnection. Hence, by virtue of having two ion energy discontinuities, this example is very helpful if we wish to 


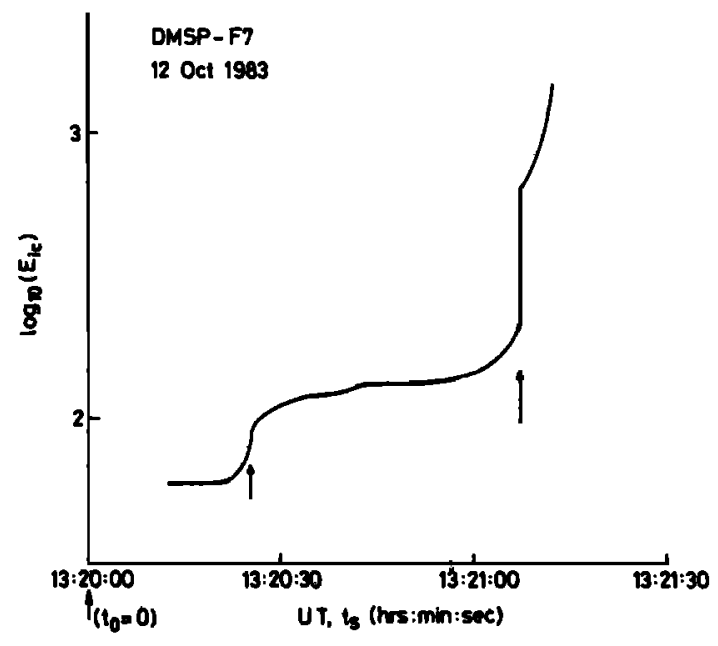

Fig. 2. The lower cutoff energy, $E_{i c}$, of cusp ions observed by the DMSP F7 satellite on October 12,1983, as presented by Newell and Meng [1991]. The arrows mark the jumps in ion energy which are discussed in detail in the text.

investigate any periodicity in the merging gap electric field $E_{y}$ and the magnetopause reconnection rate $E_{y}^{\prime}$.

Close inspection of this and all other cusp spectrograms reveals that the minimum $E_{\|}$(the lower cutoff energy $E_{i c}$ ) is not easy to define. This is because scattering ensures that there is some flux at energies below the actual time-of-flight cutoff. In this section we take $E_{i c}$ to be the lower decile of the distribution of ion energy flux (i.e., where ion energy flux falls below $10 \%$ of its peak value as determined from the spectrogram) at any one observation time, $t_{s}$. This choice is somewhat arbitrary and is made to allow determination of $E_{i c}$ from the spectrogram for as great a range of $t_{s}$ as possible. A more tigorous determination of $E_{i c}$ would require a full analysis of the scattering mechanisms. We also note that the lower cutoff appears less well defined in the cusp spectrograms (which show contours of energy flux) than in contours of phase space density (energy flux divided by the square of the energy), which is the more relevant to these time-of-flight considerations. Analysis reveals that adoption of other definitions for $E_{i c}$ does not significantly alter the results obtained.

For this definition the variation of the lower cutoff ion energy, $E_{i c}$, is shown in Figure 2. The two jumps seen in the spectrum as a whole can also be clearly seen in this plot, marked by the two arrows. From Figure 2 we can estimate $E_{i c}$ and $d E_{i c} / d t_{s}$. For simplicity, the angle $\alpha$ is taken to be zero (e.g., the satellite moves north across an east-west-aligned merging gap). We will consider other values for $\alpha$ later.

For a variety of values for $d^{\prime}$, equation (3) is then used to estimate the reconnection time, $t_{0}$, and equation (7) yields the electric field at the ionospheric merging gap at that time. For convenience, 1320:00 UT is defined as $t_{0}=0$.

In order to make a crude estimate of a realistic range of $d^{\prime}$, consider the magnetospheric segment of the newly opened field line, from the magnetopause $X$ line to the ionosphere, to be semicircular at the time $t_{0}$. For a magnetopause which is compressed to within $6 \boldsymbol{R}_{E}$ of the Earth by an exceptionally large solar wind dynamic pressure, this yields a $d$ value of about $10 R_{E}$. However, for a very distant magnetopause at $20 R_{E}$, this yields $d=30 R_{E}$. In addition, we can account for any difference between $d$ and $d^{\prime}$. If we consider that (for

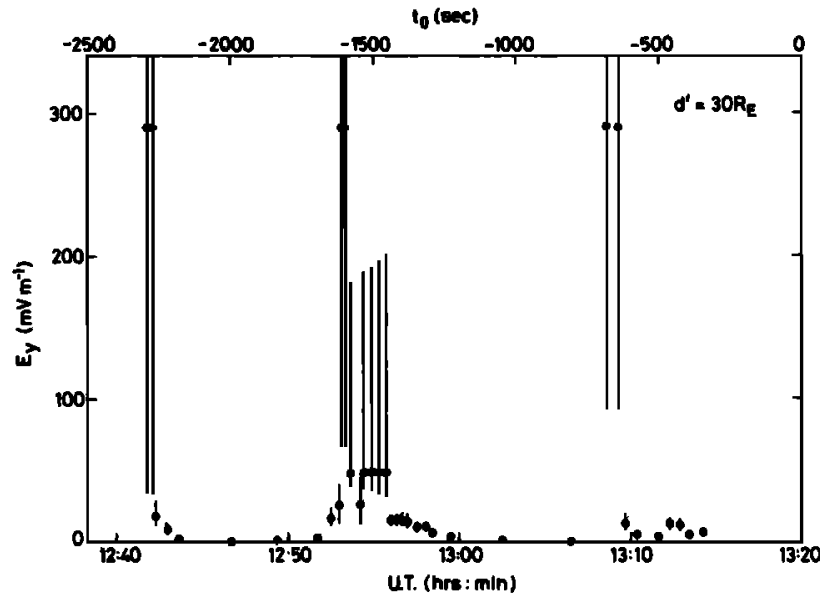

Fig. 3. Values of merging gap electric field, $E_{y}$, as a function of time. The dots are the most probable values, but the vertical error bars give the full range of possible values. In this case the "virtual" injection distance, $d^{\prime}$, is $30 R_{E}$.

example) the ion energy was doubled by low-altitude acceleration at $1 \boldsymbol{R}_{E}$ altitude to the $E_{\|}$observed by the satellite, we find $d^{\prime}$ is roughly equal to $1.5 d$. Here we shall consider $d^{\prime}$ values between $10 R_{E}$ and $50 R_{E}$, but we consider the range 15-30 $R_{E}$ to be a likely and realistic one.

Figure 3 shows values of $E_{y}$ for $d^{\prime}=30 R_{E}$ as a function of $t_{0}$ (and hence UT). The dots are the values given by the $E_{i c}$ variation plotted in Figure 2. For each point an error bar has been estimated by considering the possible error in $d E_{i c} / d t_{s}$. It will be noted that there are three peaks in $E_{y}$ but that the value at each is $290 \mathrm{mV} \mathrm{m}^{-1}$. This is because $d E_{i c} / d t_{s}$ at these times is zero (to within the accuracy with which the spectrogram can be read). At such times the uncertainty of $d E_{i c} / d t_{s}$ is such that the denominator of equation (7) could be zero, which would give a infinite $E_{y}$. Hence at the peaks of $E_{y}$ the accuracy available to us effectively only allows determination of a minimum electric field. However, in each of the bursts the minimum electric field is considerably greater (by a factor of about 100 ) than the maximum value between the bursts. Note that because few estimates of the cutoff energy and its time derivative can be made during the jumps, the number of data points is low in the minima of the electric field (which produced the jumps).

In order to understand the dependence of this result on the assumed value for $d^{\prime}$, Figure 4 is the same as Figure 3, but for $d^{\prime}$ of a smaller value of $20 R_{E}$. The three bursts in electric field are still clearly evident; indeed, the uncertainties are slightly smaller than in Figure 3. The values of $E_{y}$ are not substantially different than those in Figure 3, but the bursts occur significantly later because of the smaller times of flight. Hence the periods between the events are smaller. This is shown clearly in Figure 5, which plots the interval between the first and second peaks in $E_{y}, \Delta t_{12}$, and between the second and third peak, $\Delta t_{23}$, as a function of $d^{\prime}$. The period $\Delta t_{12}$ can be seen to increase from $4 \mathrm{~min}$ for the small $d^{\prime}$ of $10 R_{E}$ to $19 \mathrm{~min}$ for the very large value for $d^{\prime}$ of $50 R_{E}$, while $\Delta t_{23}$ behaves similarly, increasing from $5.5 \mathrm{~min}$ to 26 min for the same range of $d^{\prime}$.

\section{Discussion}

The satellite pass described here is northward, almost along a magnetic meridian. Hence the merging gap would 


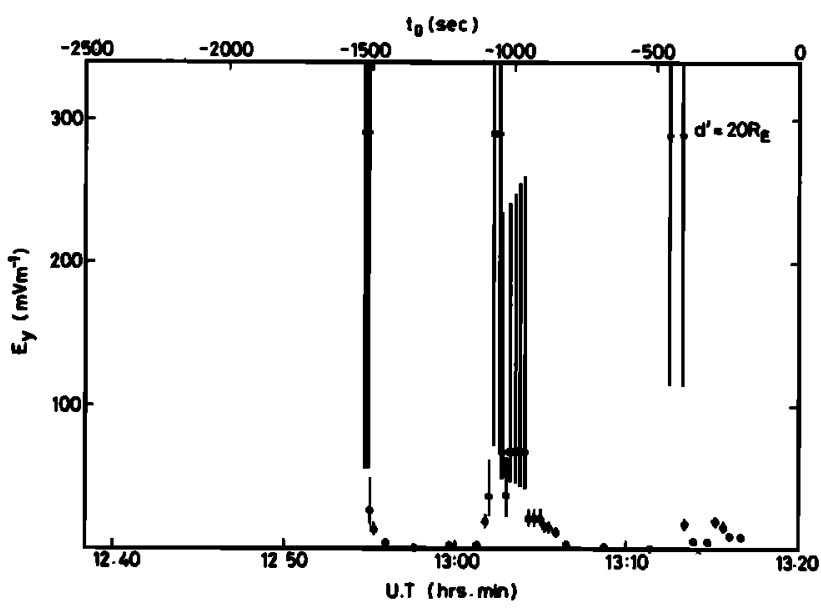

Fig. 4. Same as Figure 3, but for $d^{\prime}$ of $20 R_{E}$.

have to be at a large angle to the $L$ shells for our assumed value of $0^{\circ}$ for the angle $\alpha$ to be seriously in error. More importantly, it seems highly unlikely that this angle could vary by such a large factor on minute time scales, such that it could explain the increases in $E_{y}$ shown in Figures 3 and 4. Likewise sufficient variations of the geometrical factor $d y /$ $d y^{\prime}$ seem unlikely. This factor depends upon the amount on newly opened flux which threads the dayside magnetopause. Such flux acts to "open" the cusp; i.e., it increases its longitudinal extent [Crooker et al., 1991; Lockwood, $1991 b$ ]. The flux connected in the short bursts shown in Figures 3 and 4 is not sufficient to substantially alter this factor. This being the case, the most likely explanation is that the derived electric field variations reflect variations in the reconnection rate at the dayside magnetopause, $E_{y}^{\prime}$.

The inferred variation of the reconnection rate is very similar to that required to explain the characteristic magnetopause signatures called flux transfer events (FTEs) [Russell and Elphic, 1978]. These signatures are known to recur on time scales of $8 \mathrm{~min}$ on average when the IMF is consistently southward, although there is considerable variability about this figure [Rijnbeek et al., 1984; Berchem and

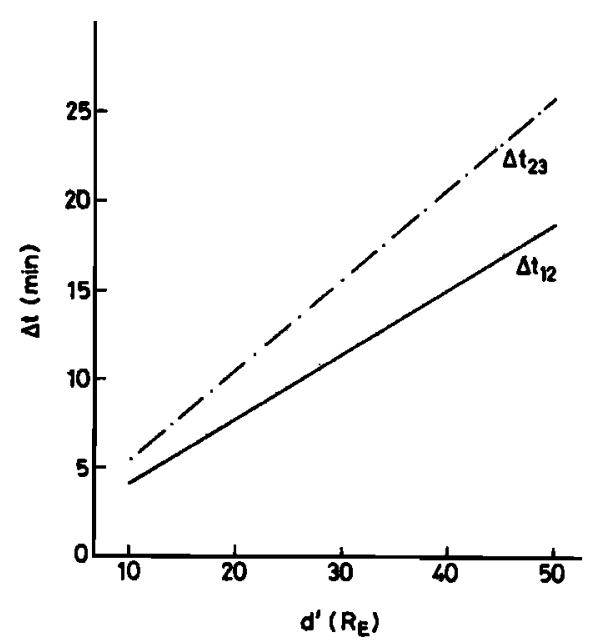

Fig. 5. The variation of the intervals between the onsets of the first and second and the second and third bursts of reconnection, $\Delta t_{12}$ and $\Delta t_{23}$, respectively, as a function of $d^{\prime}$.
Russell, 1984; Elphic, 1990]. For the realistic range of $d^{\prime}$ of 15-30 $R_{E}$, Figure 5 yields periods of 6-16 min, which is certainly consistent with FTE behavior. In addition, FTE signatures typically last for $1 \mathrm{~min}$. If the bubble containing the loops of newly opened flux has not expanded since being reconnected, the Southwood et al./Scholer model calls for a burst in the reconnection rate which is also of about $1 \mathrm{~min}$ duration. Again, this is broadly consistent with the duration of the bursts in Figures 3 and 4.

This one case certainly does not prove that the cusp precipitations (between the jumps) are FTE signatures. Probably the best way to prove such a relationship is to apply the method described here to cusp observations during periods when another satellite was close to the magnetopause (and the IMF was southward). However, one can state that the Southwood et al./Scholer FTE model predicts that the reconnection rate variation implied by Figures 3 and 4 would give three FTE signatures on the magnetopause. Consequently, it is reasonable to state that the results presented here are highly suggestive of FTE signatures.

We propose, therefore, that the presence of jumps in the cusp spectrogram is indicative of time-dependent reconnection at the dayside magnetopause, which is also expected to be the source of the FTE signatures observed there. Howèver, it should be noted that the FTE signatures in the ionosphere (i.e., the signatures of the burst of enhanced reconnection) are not the jumps, but the patches of cusp precipitation between them. Hence the analysis presented here is consistent in spirit with the suggestion by Lockwood and Smith [1989, 1990] that the cusp is an FTE signature. In fact, the cusp in the case described here is made up of three contiguous signatures of successive FTEs. Indeed, inspection of the cusp observation by the DE 2 satellite which Lockwood and Smith [1989] discussed reveals a jump in the lower cutoff energy of the ions at the equatorward edge of what the authors interpret to be the FTE signature. This jump is very similar to that discussed here.

It is interesting to study the implications of these signatures for ionospheric convection. To do this, consider that the merging gap is $1500 \mathrm{~km}$ in length, consistent with the statistical cusp width for southward IMF [Newell and Meng, 1988; Newell et al., 1989]. If we assume that the electric field along a merging gap of this length is constant and that the values given by the dots in Figures 3 and 4 are correct, we find that the reconnection voltage during each of the bursts is very high (435 kV). The electric field value corresponds to plasma crossing the $X$ line projection (in its own rest frame) at $5 \mathrm{~km} \mathrm{~s}^{-1}$. This is a very large plasma flow if the merging gap is still in the Earth's frame, but would be reduced if the merging gap moved equatorward during the reconnection burst. If we consider $d^{\prime}=20 R_{E}$, for example, we find that at least $1.9 \times 10^{7} \mathrm{~Wb}$ of flux is opened during the first burst, $2.1 \times 10^{7} \mathrm{~Wb}$ in the second, and $3.0 \times 10^{7} \mathrm{~Wb}$ in the third. Hence the three bursts open a total of $7 \times 10^{7} \mathrm{~Wb}$, out of the total of $8 \times 10^{7} \mathrm{~Wb}$ reconnected during the $1380 \mathrm{~s}$ for which $E_{y}$ can be determined for this $d^{\prime}$. Hence the average voltage during this period is $8 \times 10^{7} / 1380=58 \mathrm{kV}$, but $87.5 \%$ of this comes from three brief bursts when the voltage reached 435 $\mathrm{kV}$. All the voltages would be scaled up (down) proportionally if the merging gap length were in fact larger (smaller) than the $1500 \mathrm{~km}$ assumed here, but would decrease as $\cos \alpha$ if the angle $\alpha$ increases from zero. In addition, all values could be a factor of about 6 lower, within the experimental uncer- 
tainties plotted in Figures 3 and 4. However, the magnitude of the peaks in $E_{y}$ and the mark/space ratio of its pulsed variation are almost independent of $d^{\prime}$. As a result, the voltages derived here are hardly influenced at all by the choice of $d^{\prime}$.

The variation of the reconnection voltage inferred above is very similar to that deduced by Lockwood et al. [1990b] for the ground-based observations of dayside auroral transients and associated plasma flow bursts. Both the radar and optical measurements of the spatial extent of nine such events show that they contain magnetic fluxes between about $0.7 \times 10^{7} \mathrm{~Wb}$ and $3.4 \times 10^{7} \mathrm{~Wb}$, with typical values being about $2.5 \times 10^{7}$. This value is comparable with that deduced above assuming a $1500-\mathrm{km}$ merging gap which is the typical east-west extent of the events observed from the ground. Lockwood et al. assumed that each event was reconnected in a burst lasting $2 \mathrm{~min}$, which is roughly twice as long as derived here; hence their estimate of the reconnection voltage in the bursts was roughly half that derived here. However, the average voltage for the period is not dependent on the assumed length of the reconnection burst, and hence the value of $30 \mathrm{kV}$ derived by Lockwood et al. can be compared to the $56 \mathrm{kV}$ inferred here from the cusp precipitation.

It is also interesting to consider the implications for the reconnection electric field tangential to the magnetopause. If the length mapping factor between the $X$ line and the merging gap $\left(d y / d y^{\prime}\right)$ can be estimated, the reconnection rate at the $X$ line can be calculated. If we take a crude estimate that the field just inside the dayside magnetopause is $\left(B_{i} / 1000\right)=50 \mathrm{nT}$, flux tubes of symmetrical cross section give $\left(d y^{\prime} / d y\right) \approx 35$, and the assumed merging gap length would correspond to an $\mathrm{X}$ line of about $8 R_{E}$. This being the case, the reconnection voltage of $435 \mathrm{kV}$ in the bursts would call for a tangential electric field of $8.5 \mathrm{mV}$ $\mathrm{m}^{-1}$. However, we should add that we do not believe that any current magnetic field model can be used to give an accurate estimate of this mapping factor. This is because it will depend critically upon the amount of open flux which threads the dayside magnetopause and hence upon the recent history of the IMF $B_{z}$ component. This "opening" of the cusp by dayside open flux (i.e., the fact that $d y / d y^{\prime}$ increases following a southward turning of the IMF) has been modeled by Crooker et al. [1991], and the implications have been discussed by Lockwood [1991b].

\section{CONCLUSIONS}

A method for determining the variation of the electric field in the ionospheric merging gap (in its own rest frame) from cusp ion precipitation characteristics has been described. The orientation of the merging gap relative to the satellite orbit must be assumed, but if this is constant the electric field values derived simply vary as the sine of the angle between them. In addition, a value for the "virtual injection distance," $d$ ', is required. This will be approximately the same as the distance between the reconnection $X$ line and the satellite. The chief effects of $d^{\prime}$ are to change the time at which a reconnection burst is predicted to occur and to alter the interval between features in the electric field variation.

If information about the longitudinal extent of the merging gap in the ionosphere can be obtained, then the voltage across the merging gap (which by definition equals the voltage along the magnetopause $X$ line) can be estimated. This assumes that the electric field along the merging gap is constant. The merging gap extent could be determined by ground-based radar and optical measurements of the transient events which would result from the variations in the reconnection rate. Alternatively, if the geometrical field line mapping factor from the magnetopause $\mathrm{X}$ line to the merging gap can be estimated, then the magnetopause reconnection rate can be computed.

The method reveals very large variations in the merging gap electric field for a satellite pass which shows major jumps in the ion dispersion characteristics. The reconnection is found to occur almost exclusively in bursts about $1 \mathrm{~min}$ long and which repeat on time scales of the order of $10 \mathrm{~min}$. However, the precise value of these durations and intervals is dependent on the assumed value for $d^{\prime}$. This behavior is exactly that required to explain typical magnetopause flux transfer event signatures.

The chief uncertainty in the method lies in the definition of the lower cutoff energy and hence in its time derivative. Often this results in the method only defining a lower limit for the merging gap electric field when it is very large. However, small electric field values are determined with high accuracy. Higher-resolution measurements of the ion energy dispersion characteristics would allow this uncertainty to be reduced considerably.

If we assume a typical merging gap extent of $1500 \mathrm{~km}$ for the one event studied here, consistent with the statistical cusp width and the size of the dayside auroral and flow transients, we find that the average dayside reconnection voltage is about $60 \mathrm{kV}$ but that almost $90 \%$ of this is contributed by the bursts of reconnection during which the reconnection voltage rises to over $400 \mathrm{kV}$. The magnetopause electric field would then be about $8 \mathrm{mV} \mathrm{m}^{-1}$ during the bursts. Taking the absolute minimum values defined by the technique, these voltages and electric fields are all reduced by a factor of about 6 . Hence, even if we take the minimum values for the reconnection rate during the bursts shown in Figures 3 and 4, the results are surprising.

Lastly, the question arises as to how common are the reconnection rate pulses which are inferred in this paper (for just one satellite pass). This requires a full survey of a large amount of cusp data, using the method described in this paper. However, the problem is complex, and a simple statistical survey of the occurrence of ion energy jumps, of the kind described here, must take these complexities into account. For example, Smith et al. [1992] have recently used a simple model to study the probability of observing the jumps in the ion spectrogram, in the presence of discrete reconnection events recurring every $8 \mathrm{~min}$ (the average repetition rate of FTEs). These authors point out that the probability of observing a jump is greatly reduced by the presence of a large $B_{y}$ component of the IMF, giving a tension force which moves events either east or west. Furthermore, many of the jumps will be smaller than in the example studied here and hence harder to detect.

Measurement of the reconnection electric field along the $\mathrm{X}$ line on the dayside magnetopause (in its own rest frame) or along the dayside ionospheric merging gap has been an important but elusive goal for many years. The method presented here provides a novel way of studying the variation of these electric fields. The results indicate that reconnection can take place in a series of bursts, exactly as required to explain magnetopause flux transfer events. 
Acknowledgments. Some of this work was supported by NASA grant NAGW-1638. Many of the ideas for this paper were stimulated by the GEM workshop held at Northeastern University, October 1990. In addition, we wish to thank S. W. H. Cowley and P. T. Newell for many valuable discussions.

The Editor thanks the two referees for their assistance in evaluating this paper.

\section{REFERENCES}

Berchem, J., and C. T. Russell, Flux transfer events on the magnetopause: Spatial distribution and controlling factors, $J$. Geophys. Res., 89, 6689-6703, 1984.

Cowley, S. W. H., The causes of convection in the Earth's magnetosphere: A review of developments during IMS, Rev. Geophys., 20, 531-565, 1982.

Cowley, S. W. H., and M. Lockwood, Excitation and decay of solar wind-driven flows in the magnetopause-ionosphere system, $A n n$. Geophys., 10, 103-115, 1992.

Cowley, S. W. H., J. P. Morelli, and M. Lockwood, Dependence of convective flows and particle precipitation in the high-latitude dayside ionosphere on the $X$ and $Y$ components of the interplanetary magnetic field, J. Geophys. Res., 96, 5557-5564, 1991a.

Cowley, S. W. H., M. P. Freeman, M. Lockwood, and M. F. Smith, The ionospheric signature of flux transfer events, in CLUSTERDayside Polar Cusp, edited by C. I. Barron, Eur. Space Agency Spec. Publ., ESA SP-330, 105-112, 19916.

Crooker, N. U., F. R. Toffoletto, and M. S. Gussenhoven, Opening the cusp, J. Geophys. Res., 96, 3497-3503, 1991.

Daly, P. W., M. A. Saunders, R. P. Rijnbeek, N. Sckopke, and C. T. Russell, The distribution of reconnection geometry in flux transfer events using energetic ion, plasma and magnetic data, $J$. Geophys. Res., 89, 3843-3854, 1984.

Elphic, R. C., Observations of flux transfer events: Are FTEs flux ropes, islands, or surface waves?, in Physics of Magnetic Flux Ropes, Geophys. Monogr. Ser., vol. 58, edited by C. T. Russell, E. R. Priest, and L. C. Lee, pp. 455-472, AGU, Washington, D. C., 1990.

Farrugia, C. J., R. C. Elphic, D. J. Southwood, and S. W. H. Cowley, Field and flow perturbations outside the reconnected field line region in flux transfer events: Theory, Planet. Space Sci., 35, 227-240, 1987a.

Farrugia, C. J., D. J. Southwood, S. W. H. Cowley, R. P. Rijnbeek, and P. W. Daly, Two-regime flux transfer events, Planet. Space Sci., 35, 737-746, 1987b.

Farrugia, C. J., R. P. Rijnbeek, M. A. Saunders, D. J. Southwood, D. J. Rodgers, M. F. Smith, C. P. Chaloner, D. S. Hall, P. J. Christiansen, and L. J. C. Woolliscroft, A multi-instrument study of flux transfer event structure, J. Geophys. Res., 93, 14,465$14,477,1988$

Fuselier, S. A., D. M. Klumpar, and E. G. Shelley, Ion reflection and transmission during reconnection at the Earth's subsolar magnetopause, Geophys. Res. Lett., 18, 139-142, 1991

Gosling, J. T., M. F. Thomsen, S. J. Bame, R. C. Elphic, and C. T. Russell, Plasma flow reversals at the dayside magnetopause and the origin of asymmetric polar cap convection, J. Geophys. Res., $95,8073-8084,1990$.

Haerendel, G., G. Paschmann, N. Sckopke, H. Rosenbauer, and P. C. Hedgecock, The frontside boundary layer of the magnetopause and the problem of reconnection, J. Geophys. Res., 83, $3195-3216,1978$

Hill, T. W., Rates of mass, momentum, and energy transfer at the magnetopause, in Proceedings of the Magnetospheric Boundary Layers Conference, Alpbach, Eur. Space Agency Spec. Publ., ESA SP-148, 325-333, 1979.

Lee, L. C., and Z. F. Fu, A theory of magnetic flux transfer at the Earth's magnetopause, Geophys. Res. Lett., 12, 105-108, 1985.

Lockwood, M., Flux transfer events at the dayside magnetopause: Transient reconnection or magnetosheath pressure pulses?, $J$. Geophys. Res., 96, 5497-5509, 1991a.

Lockwood, M., Incoherent scatter radar measurements of the cusp, in CLUSTER-Dayside Polar Cusp, edited by C. I. Barron, Eur. Space Agency, Spec. Publ., ESA SP-330, 57-66, 1991 b.

Lockwood, M., and M. F. Smith, Low altitude signatures of the cusp and flux transfer events, Geophys. Res. Lett., 16, 879-882, 1989.
Lockwood, M., and M. F. Smith, Reply to Newell, Geophys. Res. Lett., 17, 305-306, 1990.

Lockwood, M., S. W. H. Cowley, and M. P. Freeman, The excitation of plasma convection in the high-latitude ionosphere, $J$ Geophys. Res., 95, 7961-7971, 1990a.

Lockwood, M., S. W. H. Cowley, P. E. Sandholt, and R. P. Lepping, The ionospheric signatures of flux transfer events and solar wind dynamic pressure changes, J. Geophys. Res., 95, $17,113-17,135,1990 b$.

Menietti, J. D., and J. L. Burch, Spatial extent of the plasma injection region in the cusp-magnetosheath interface, J. Geophys. Res., 93, 105-113, 1988.

Newell, P. T., Comment, Geophys. Res. Lett., 17, 303-304, 1990.

Newell, P. T., and C.-I. Meng, The cusp and the cleft/boundary layer: Low-altitude identification and statistical local time variation, J. Geophys. Res., 93, 14,549-14,556, 1988.

Newell, P. T., and C.-I. Meng, Ion acceleration at the equatorward edge of the cusp: Low altitude observations of patchy merging, Geophys. Res. Lett., 18, 1829-1832, 1991.

Newell, P. T., C.-I. Meng, D. G. Sibeck, and R. Lepping, Some low-altitude cusp dependencies on the interplanetary magnetic field, J. Geophys. Res., 94, 8921-8927, 1989.

Paschmann, G., G. Haerendel, I. Papamastorakis, N. Sckopke, S. J. Bame, J. T. Gosling, and C. T. Russell, Plasma and magnetic field characteristics of magnetic flux transfer events, J. Geophys. Res., 87, 2159-2168, 1982.

Reiff, P. H., T. W. Hill, and J. L. Burch, Solar wind plasma injection at the dayside magnetospheric cusp, J. Geophys. Res., 82, 479-491, 1977.

Rijnbeek, R. P., S. W. H. Cowley, D. J. Southwood, and C. T. Russell, A survey of dayside flux transfer events observed by the ISEE 1 and 2 magnetometers, J. Geophys. Res., 89, 786-800, 1984.

Rosenbauer, H., H. Grünwaldt, M. D. Montgomery, G. Paschmann, and N. Sckopke, HEOS 2 plasma observations in the distant polar magnetosphere: The plasma mantle, J. Geophys. Res., 80, 2723-2737, 1975.

Russell, C. T., and R. C. Elphic, Initial ISEE magnetometer results: Magnetopause observations, Space Sci. Rev., 22, 681-715, 1978.

Russell, C. T., and R. C. Elphic, ISEE observations of flux transfer events at the dayside magnetopause, Geophys. Res. Lett., 6, 33-36, 1979.

Scholer, M., Magnetic flux transfer at the magnetopause based on single X-line bursty reconnection, Geophys. Res. Lett., 15, 291294, 1988.

Scudder, J. D., K. W. Ogilvie, and C. T. Russell, The relation of flux transfer events to magnetic reconnection, in Magnetic Reconnection in Space and Laboratory Plasmas, Geophys. Monogr. Ser., vol. 30, edited by E. W. Hones, Jr., p. 151, AGU, Washington, D. C., 1984

Smith, M. F., and M. Lockwood, The pulsating cusp, Geophys. Res. Lett., 17, 1069-1072, 1990.

Smith, M. F., and D. J. Rodgers, Ion distributions at the dayside magnetopause, J. Geophys. Res., 96, 11,617-11,624, 1991.

Smith, M. F., M. Lockwood, and S. W. H. Cowley, The statistical cusp: A flux transfer event model, Planet. Space Sci., 40, 1251-1268, 1992.

Southwood, D. J., Theoretical aspects of ionosphere, magnetosphere-solar wind coupling, Adv. Space Res., 5(4), 7-14, 1985.

Southwood, D. J., The ionospheric signature of flux transfer events, J. Geophys. Res., 92, 3207-3213, 1987.

Southwood, D. J., M. A. Saunders, M. W. Dunlop, W. A. C. Mier-Jedrzejowicz, and R. P. Rijnbeek, A survey of flux transfer events recorded by UKS spacecraft magnetometer, Planet. Space Sci., 34, 1349-1359, 1986.

Southwood, D. J., C. J. Farrugia, and M. A. Saunders, What are flux transfer events?, Planet. Space Sci., 36, 503-508, 1988.

M. Lockwood, Rutherford Appleton Laboratory, Space Science Department, R25, room 1-04, Chilton, Didcot, Oxon OX11 OQX, England.

M. F. Smith, NASA Goddard Space Flight Center, Code 696, Greenbelt, MD 20771.

(Received February 11, 1992; revised June 17, 1992; accepted May 22, 1992.) 